\title{
Observation of Strongly Heterogeneous Dynamics at the Depinning Transition in a Colloidal Glass
}

\author{
Nesrin Senbil, ${ }^{1, \dagger}$ Markus Gruber, ${ }^{2, \dagger}$ Chi Zhang, ${ }^{1}$ Matthias Fuchs, ${ }^{2}$ and Frank Scheffold ${ }^{1, *}$ \\ ${ }^{1}$ Department of Physics, University of Fribourg, CH-1700 Fribourg, Switzerland \\ ${ }^{2}$ Fachbereich Physik, Universität Konstanz, 78457 Konstanz, Germany
}

(Received 16 August 2018; revised manuscript received 6 December 2018; published 13 March 2019)

\begin{abstract}
We study experimentally the origin of heterogeneous dynamics in strongly driven glass-forming systems. Thereto, we apply a well-defined force with a laser line trap on individual colloidal polystyrene probe particles seeded in an emulsion glass composed of droplets of the same size. Fluid and glass states can be probed. We monitor the trajectories of the probe and measure displacements and their distributions. Our experiments reveal intermittent dynamics around a depinning transition at a threshold force. For smaller forces, linear response connects mean displacement, and quiescent mean squared displacement. Mode coupling theory calculations rationalize the observations.
\end{abstract}

DOI: $10.1103 /$ PhysRevLett.122.108002

Tracking the passive or driven motion of a colloidal probe particle immersed in a complex environment, known as microrheology, provides unique insights into the local mechanical and transport properties of materials [1-3]. Individual probe trajectories can be recorded and the heterogeneity of the dynamics can be studied directly [3-5]. In active microrheology the motion of driven tracer particles is analyzed to probe the systems dynamics [3]. Yet, it is often unclear if and when the probe faithfully samples the intrinsic thermal motion, especially at strong driving. Experimental probe trajectories, e.g., in living cells [6], often shown deviations from classical drift-diffusive motion [7].

Earlier experimental work explored the linear and nonlinear regimes in colloidal model systems [8,9]. In their seminal work, Habdas et al. studied the nonlinear force to average velocity relations [8]. Yet, experimental studies of the predicted, highly anomalous, spatiotemporal distributions of probe displacements are still lacking. Computer simulations suggest that probability distribution functions of the probe displacements in glassy systems are anomalously broad [10-14], generally non-Gaussian, and often bimodal. The existence of two subpopulations of probes, one of which remains stuck in the glassy surroundings for long times, while the other moves (far) in the direction of the force, has been discovered in simulations of supercooled liquids $[13,14]$ and of active particle systems [15]. Bimodality has been observed for short times in the motion of colloids in corrugated tracks [16], while power-law distributions are observed in granular systems close to jamming [11,17]. Hydrodynamic models [18], mesoscopic models of glassy dynamics, like trap [19] and continuous time random walk models [20-22], and lattice models of transport in random media $[23,24]$ have shown that the splitting into two populations lies at the origin of the intermittency in the probe motion. Microscopic mode coupling theory has identified a threshold force for the delocalization (depinning) of a probe particle in glass $[12,25]$; average motion only sets in for forces larger than the threshold.

Here we show experimentally and theoretically that structurally homogeneous colloidal suspensions around the glass transition exhibit heterogeneous and intermittent dynamics when a particle is driven by an external force. Our experiments are performed on a near-ideal model system for hard spheres, which displays only weak dynamic heterogeneities in the quiescent state $[26,27]$. Beyond a threshold force we observe strongly intermittent dynamics and bimodal van Hove distribution functions. For smaller forces, linear response connects the particle mean displacement and quiescent mean squared displacement. Using results from mode coupling theory we can rationalize the observations. Our findings highlight the important differences between quiescent and driven motion in crowded environments.

Experiment.-We study experimentally the active microrheology of uniform oil-in-water emulsion droplets, mean diameter $d=2.01 \mu \mathrm{m}$, that show nearly hard-sphere behavior with an experimentally confirmed glass, and a jamming transition at volume fractions of $\phi \simeq 0.59$ and 0.64 [27], respectively. For the volume fractions considered, $0.53<\phi<0.61$, the hard-spherelike droplets are far from touching and there is no stress-bearing network of contact points as is present in jammed emulsions [28-30]. The solvent and the emulsion droplets are refractive index and buoyancy matched and a small amount of added polystyrene probe particles of the same size provide optical contrast for laser trapping, see also section "Sample perparation protocol" in the Supplemental Material [31], which includes Refs. [32-34]. For each packing fraction, 
the probe particle mean square displacement (MSD) is first monitored in the quiescent state, without applying any force for $1200 \mathrm{~s}$. We find the well-known slowing down of the long-time diffusion approaching the colloidal glass transition and the caging of particles for $\phi$ above it [35]. The results are in quantitative agreement with previous experiments on similar systems [27,36] and with calculations from mode coupling theory (see Fig. S2 in the Supplemental Material [31], which includes Refs. [37]). The latter comparison confirms the mapping of short time diffusion coefficient $D_{0}$, density and length scale between measurements and theory. We position the probe particle in a gradient intensity line trap such that a constant force is created along the scan direction, while in the two perpendicular directions the particle motion is strongly confined, Fig. 1 [38]. From reference measurements in a simple viscous liquid we find that the force is constant $\pm 2 \%$ over a range of $25 \mu \mathrm{m}$, corresponding to more than 12 particle diameters, see the Supplemental Material [31], which includes Refs. $[39,40]$. The magnitude of the force can be adjusted by tuning the power of the laser $P_{l}$ and the forces that can be generated are of the order of several hundred femtonewtons. In the experiment the probe particle is first captured at a depth $z=(2 \sim 6) d$ using a (a)

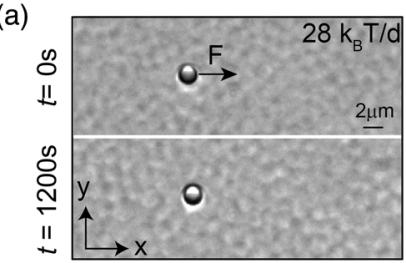

(c)

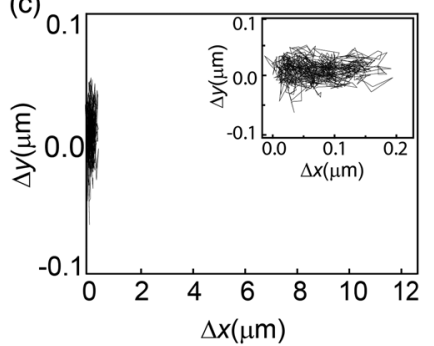

(b)

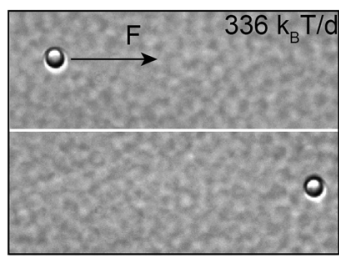

(d)

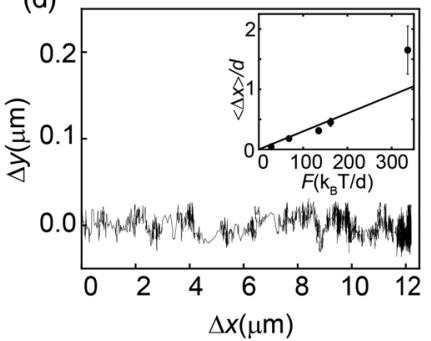

FIG. 1. Motion of a polystyrene probe particle seeded in a glassy emulsion at $\phi=0.601$. The diameter of the probe particle and the emulsion droplets $d=2 \mu \mathrm{m}$ are the same. The optical force is applied in the $x$ direction and the results for two different laser power settings are shown. (a) $F=28 k_{B} T / d$ and (b) $F=336 k_{B} T / d$ with $k_{B} T / d=2.03 \mathrm{fN}$. Probe position shown right before (top) and $20 \mathrm{~min}$ (bottom) after the constant force line trap has been activated. The lower panels (c),(d) show a map of $x-y$ positions of the probe particle over the whole duration of the experiment. Corresponding movies (accelerated 10×) are included in the Supplemental Material [31]. Inset (c): Enlarged view of the probe particle trajectory. Inset (d): Average probe displacement at $60 \mathrm{~s}$ at each force. Solid line shows the linear response law Eq. (1) using the measured MSD at $60 \mathrm{~s}$ times the applied forces single-beam point trap. Larger depths are not accessible due to residual scattering and optical aberrations. The finite depth might induce a small numerical shift of the results due to wall effects, similar to the case of simple fluids [41], but we do not expect significant qualitative changes of the dynamics. Subsequently, we align the optical tweezer and the probe particle position, and at $t=t_{0}$ switch the optical configuration to apply a constant force $\vec{F}\left(t>t_{0}\right)=F \vec{e}_{x}$ in the $x$ direction parallel to the surface of the sample. The image acquisition is started at a time $t$ with a delay of $t-t_{0}=0-0.2 \mathrm{~s}$ for the smaller and $t-t_{0} \sim 0.5-1 \mathrm{~s}$ for higher forces, the latter due to an earlier realization of the experiment. The accuracy of tracking the probe particle is approximately $\pm 30 \mathrm{~nm}$ [27]. The main uncertainty arises from the unknown delay $t-t_{0}$. We take account of this by plotting a systematic error interval, as shown in Fig. 2 (there and in all following plots $t_{0}=0$ is set). Data points for larger displacements and longer times, which are our main interest, are not affected due to the logarithmic scales employed. Using a digital camera we record five images of the sample per second and subsequently track the position of the particle for each frame using standard procedures [3]. For each force the experiment is repeated more than 10 times on different probe particles and in some cases up to 40 times.

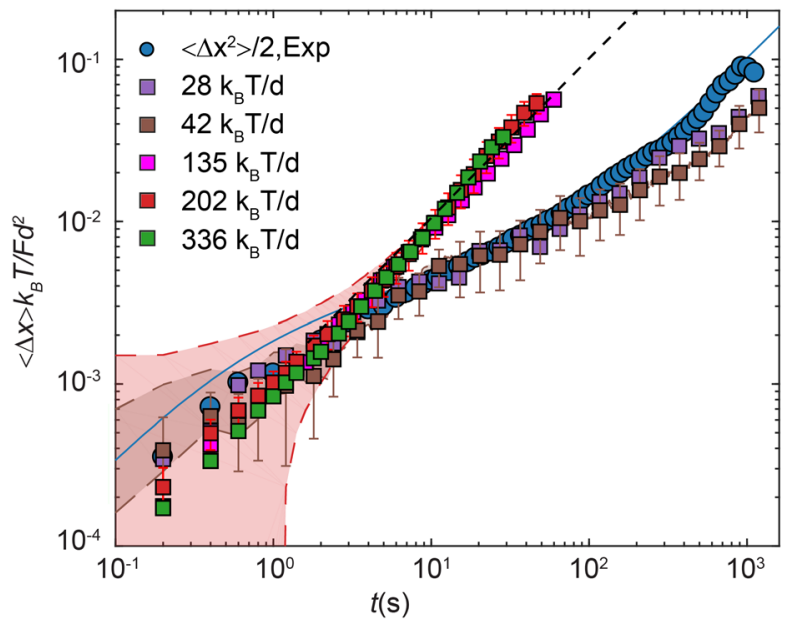

FIG. 2. Mean motion of the probe in a supercooled liquid: The rescaled mean displacement (MD) $\langle\Delta x(t)\rangle / F$ at $\phi=0.535$ are shown as squares for different forces as labeled in $k_{B} T / d$. Overlap with the quiescent MSD curve (in units of $d^{2}$, blue circles) and MCT prediction for the MSD (solid line) for the lower forces verifies the validity of linear response, Eq. (1). For the higher forces the dashed line marks the linear drift regime with $\langle\Delta x(t)\rangle /\left(F d^{2} / k_{B} T\right) \simeq 10^{-3} t / \mathrm{s}$. The shaded area marks the systematic error due to the uncertainty with respect to $t-t_{0}$ for the higher forces (red) and for lower forces (brown). The range of uncertainty is $[t, t+\delta t]$ and $[\Delta x(t), \Delta x(t+\delta t)]$ with $\delta t=0.2(1) \mathrm{s}$ for the lower (higher) forces. We estimate $\Delta x(t+\delta t)$ using Eq. (1). Error bars mark the statistical errors. 
Linear response and intermittent dynamics in the fluid state.-The motion of the probe particle subject to the external force will depend on the strength of the forces and the emulsion concentration. We carefully analyze the particle trajectories for two compositions, one in the viscoelastic fluid regime $(\phi=0.535)$ and one in the glass $(\phi=0.601)$. When applying a relatively small force the mean displacement (MD) of the probe should obey the linear response relation [42]:

$$
\langle\Delta x(t)\rangle=\frac{\left\langle\Delta x^{2}(t)\right\rangle_{\mathrm{qe}}}{2 k_{B} T} F
$$

which identifies the quiescent 1D-MSD $\left\langle\Delta x^{2}(t)\right\rangle_{\text {qe }}$ divided by $2 k_{B} T$ as time-integrated mobility. Equation (1) predicts that the ratio $\langle\Delta x(t)\rangle / F$ collapses onto the MSD (in units of $\left.2 k_{B} T\right)$ for times and forces where nonlinear effects are negligible. Interestingly, to our best knowledge, this law has never been tested experimentally for strongly correlated colloidal liquids. Figure 2 shows that for lower forces the linear response relation holds in the supercooled state for a wide window in time where the probe explores the glassy cage and its slow relaxation. Increasing the laser power and employing forces of order $100 k_{B} T / d$, the measured MD speed up at long times and approach a linear drift, Fig. 2. The force-induced escape from cages dominates relative to the one by thermal fluctuations in the viscoelastic fluid state. MCT supports these conclusions, see Fig. S5 in Supplemental Material [31]; quantitative differences exist in the magnitude of the effects.

Depinning and intermittent dynamics in the glass.Observation of linear response in the glass is challenging, because the displacements are small. While the uncertainty in establishing the starting point of the trajectory affects the MD data for short times, we still find linear response for $t=60 \mathrm{~s}$ as shown in the inset in panel (d) of Fig. 1. The line shows the prediction based on the measured force-free MSD at $60 \mathrm{~s}$, which is long enough to not suffer from the short time uncertainties and short enough to avoid problems due to a possible drift of the system. The data for all times with the full uncertainty analysis are shown in Fig. S7 in the Supplemental Material [31] and confirm that any time between 50 and $200 \mathrm{~s}$ would give the same results. MCT predicts a threshold force of $F_{c} d / k_{B} T=34.4$ in the glass $[12,25]$, which is well within the experimentally accessible range. It should be noted that this transition is quite sharp in theory; i.e., a small variation in the force causes are large variation in the behavior of the mean displacement and thus a phase diagram separating delocalized and localized regimes can be established as shown in Ref. [12]. In the experiments and previous simulations [12] this phenomenon appears over a broader range of forces. This makes it more difficult to find the threshold force in the experiment. From our data, inset Fig. 1(d), we estimate it to be $F_{c} d / k_{B} T \sim 135-300$, which is larger than the MCT prediction, and slightly larger than the simulation results [12].

We now turn our attention to the dynamics at the depinning transition. In Fig. 3, we show several trajectories $\Delta x(t)$ of the probe particle for an emulsion volume fraction of $\phi=0.601$ (glass) at a force $F \gtrsim F_{c}$ close or slightly above the depinning transition. For comparison, we include trajectories in the fluid $(\phi=0.535)$ for a similar force. Also the complete histograms for large median displacements $\langle\widetilde{\Delta x}\rangle=5.7 d$ are compared for fluid and glass sample. This value is determined by the largest median displacement measured in the glass. Clearly, the motion is far more
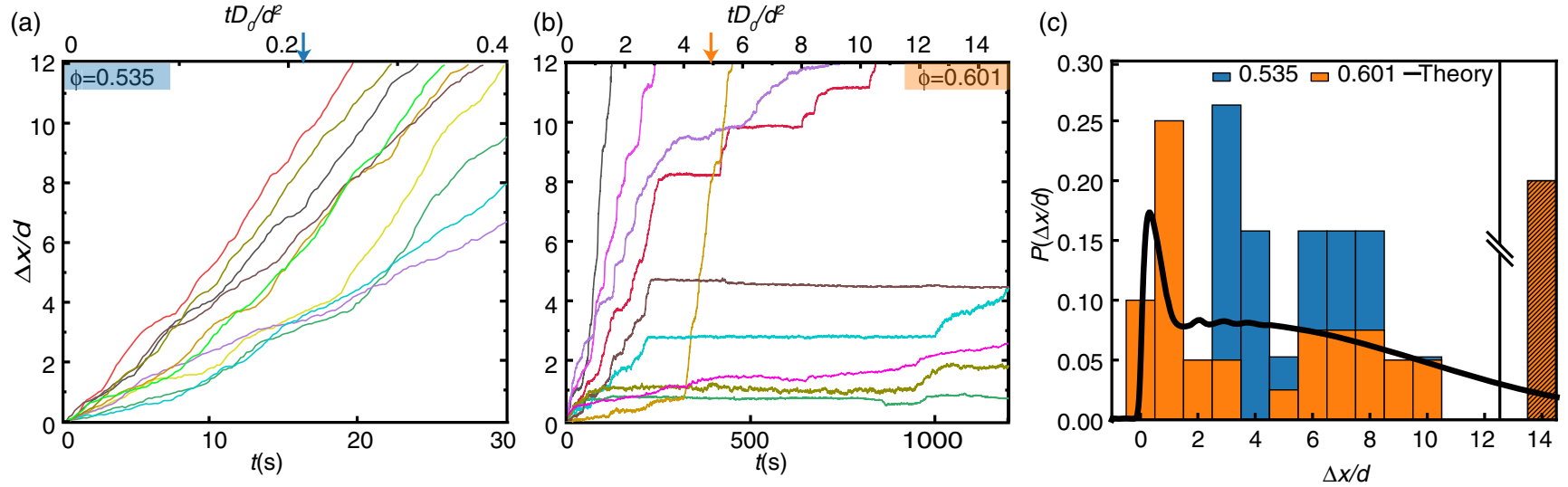

FIG. 3. Intermittent dynamics in the glass. Panels (a) and (b) show typical individual probe displacement curves in the direction of the applied force $F$ in the liquid [(a), $\left.\phi=0.535, F d / k_{B} T=336\right]$ and the glass [(b), $\left.\phi=0.601, F d / k_{B} T=336\right]$. The time window in (b) is enlarged $40 \times$ to cover the constrained probe displacements in the glass. Arrows indicate the times, at which the histograms in panel (c) were taken. Times were chosen such that the median of the data is the same $(\widetilde{\Delta x}=5.7 d)$. Particles, which have reached the end of the trap are collected in the bin at 14. To illustrate the critical behavior, we show a theory curve (solid line) at the threshold force $F_{c}=34.4 k_{B} T / d$ for a time, where the median is similar. 
intermittent in the glass than in the fluid state and the displacement distribution is far broader. The probability distribution function (PDF) of displacements in force direction, viz. the van Hove function $G^{s}(\Delta x, t)$ [3] can also be calculated from theory. MCT predicts bimodal shapes of pinned and mobilized subpopulations close to the depinning force $F_{c}$. A PDF at $F_{c}$ and identical median displacement is added for comparison in Fig. 3(c). It correlates well with the sampled histograms.

To answer the question whether the force-induced motion differs qualitatively from the (intrinsic) thermally induced particle motion, we compare the PDF of displacements for a state where thermal motion is active $(\phi=0.535)$ and one where it is not $(\phi=0.601)$. Figure 4 shows histograms of the PDF at fixed average displacement $\langle\Delta x\rangle$ comparing data at the same or comparable force settings from fluid (left) and glass (right panels) samples. The chosen distances from $\langle\Delta x\rangle=0.2 d$ (upper) to $1.6 d$ (lower panels) correspond to the largest mean displacements available in this setup. The experiment ends when the first particle reaches the end of the line trap or after around $1000 \mathrm{~s}$. We compare forces below and above the depinning transition for the glass and choose similar forces for the liquid. To illustrate the non-Gaussian behavior, a Gaussian distribution with the same average displacement (viz. MD from Fig. 2) and quiescent variance (viz. MSD at the same time) is compared to the data. Also MCT calculations for the same MD values are included. Because of the force mismatch in the theory, MCT forces are fitted to the histograms optimizing the similarity (over a range of mean displacements) following Ref. [43]. In the fluid state for the lower force, the PDF of the probe still resembles the Gaussian solution of the drift-diffusion equation expected in dilute systems [3]. In the glass at this force, however, the PDF extends to larger displacements than the shifted Gaussian even though it has the same average $\langle\Delta x\rangle=0.4 d$. The differences between the fluid and glass PDF become larger with stronger forces. In glass where force induced motion dominates, some probes remain localized within their cages, while some other probes can escape their neighborhood and reach displacements comparable to the bath particle size or larger. This reveals the heterogeneity in the cage strength and the collective origin of the force pinning the probe. MCT predicts the appearance of an exponential tail when approaching $F_{c}$ [12] which is compatible with the datahistogram albeit not clearly resolved due to the limited number of experiments $N$. Even stronger heterogeneity in the probe motion is visible at the largest forces. The interpretation suggested by theory and simulation [13] is that the PDF develops a bimodal shape in the glass consisting of one subpopulation of pinned and another subpopulation of mobilized particles. In the fluid state, the additional bath motion narrows the PDF as cages open more uniformly by thermal fluctuations. Bimodal PDF

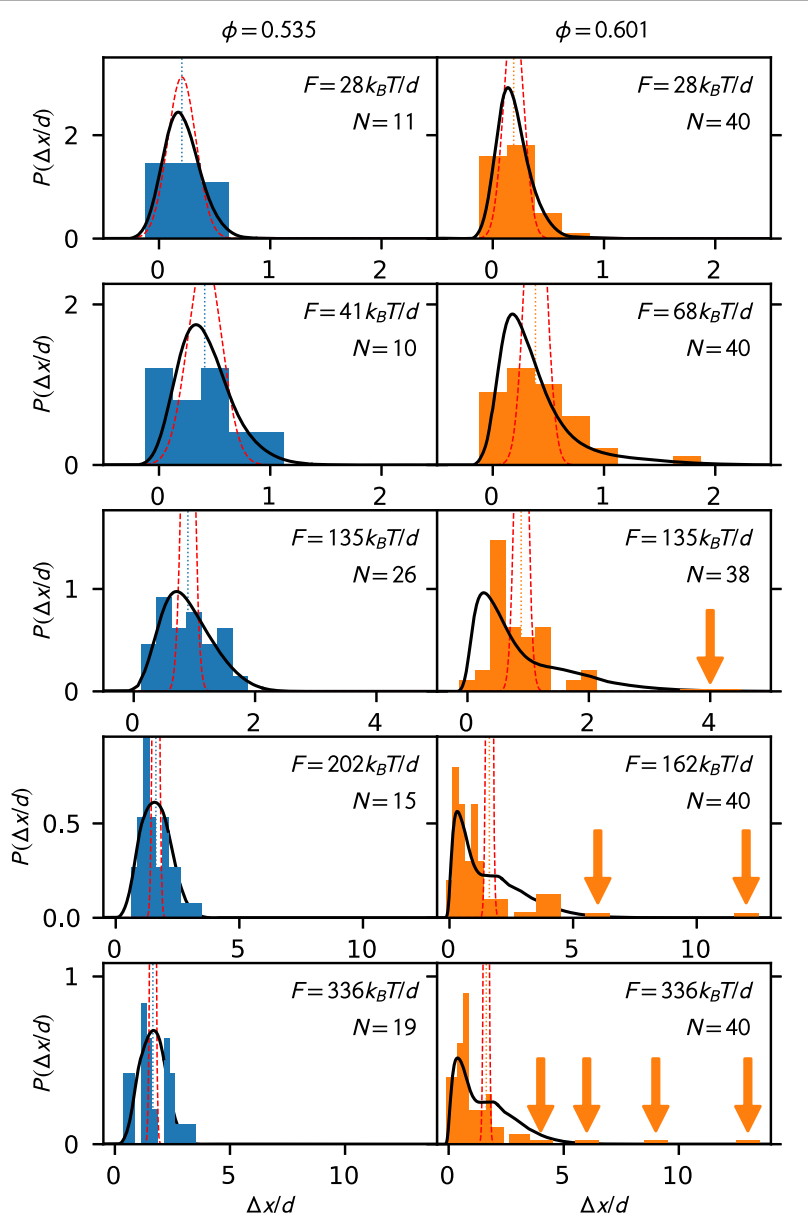

FIG. 4. Strong forces induce intermittent displacements. We compare experiments (histograms, $N$ experiments for $\phi=0.535$ and $\phi=0.601$ ) and theory (solid lines) for different applied forces at times, where the mean displacement (dotted vertical line) is the same. The left panels show the van-Hove function in the liquid ( $\phi=0.535)$, while the right panels show it in the glass $(\phi=0.601)$ for forces increasing from top to bottom. The displacements are determined from the largest displacement available in the glass: $\langle\Delta x\rangle=0.2 d, 0.4 d, 0.9 d, 1.6 d, 1.6 d$ (from top to bottom). Arrows indicate single observations. The forces in theory are chosen such that the similarity (as introduced in Ref. [43]) between the histogram and PDF is maximized. For comparison, we plot a Gaussian (dashed red line) with the same mean and variance given by the quiescent MSD at the same time. The times at which the histograms are taken are for $\phi=0.535$ : 32.2, 64.8, 7.6, 9.0, $5.8 \mathrm{~s}$ and for $\phi=0.601$ : 973, 801, 644, 642, $59 \mathrm{~s}$ (from top to bottom, i.e., low force to high forces).

arise in the MCT calculations in a range of forces below and close to the glass transition (not shown) which implies that a characteristic force remains meaningful also in fluid states; it separates intrinsic from force-induced cage breaking processes.

Discussion and conclusion.-In summary, we have shown that force-induced intermittent motion can be observed and quantified in glass-forming dispersions, tracking colloidal probes manipulated in an optical line 
trap. Linear response rationalizes the behavior for small forces of the order of $\mathcal{O}\left(10 k_{B} T / d\right)$ for a broad time window. Force-dominated motion sets in at longer times, including in glass states where a force threshold $F_{c}$ needs to be overcome. Depinning and cage breaking is characterized by intermittent probe motion and anomalous broadening of the displacement probability distribution. Theory rationalizes the observations and predicts bimodal distributions, where a subpopulation of particles remains trapped while another subpopulation moves far. Intermittent motion arises in undercooled fluid states and gets stronger when approaching the glass transition, as correlates with the growth of dynamically heterogeneous regions seen in quiescent dispersions [44]. Yet, it is strongest in glass where only smaller cooperative clusters were observed without force. This indicates that the link between intermittent motion in active microrheology and dynamical heterogeneities is more indirect than previously discussed [20,22]. A qualitative comparison with mode coupling theory is possible. In the experiment, anomalous dynamics is observed over a broader range of forces than predicted theoretically.

N. S., C. Z., and F.S. acknowledge funding by the Swiss National Science Foundation through Project No. 169074 and through the National Center of Competence in Research Bio-Inspired Materials. M. G. and M.F. acknowledge financial support from the Deutsche Forschungsgemeinschaft in Project P3 of Grant No. FOR1394. We would like to thank Veronique Trappe and Thomas G. Mason for discussions.

* Corresponding author.

frank.scheffold@unifr.ch

${ }^{\dagger}$ N. S. and M. G. contributed equally to this work.

[1] T. A. Waigh, Microrheology of complex fluids, Rep. Prog. Phys. 68, 685 (2005).

[2] T. M. Squires and T. G. Mason, Fluid mechanics of microrheology, Annu. Rev. Fluid Mech. 42, 413 (2010).

[3] E. M. Furst and T.M. Squires, Microrheology (Oxford University Press, Oxford, 2017).

[4] T. G. Mason, K. Ganesan, J. H. Van Zanten, D. Wirtz, and S. C. Kuo, Particle Tracking Microrheology of Complex Fluids, Phys. Rev. Lett. 79, 3282 (1997).

[5] S. Manley, J. M. Gillette, G. H. Patterson, H. Shroff, H. F. Hess, E. Betzig, and J. Lippincott-Schwartz, High-density mapping of single-molecule trajectories with photoactivated localization microscopy, Nat. Methods 5, 155 (2008).

[6] B. Wang, J. Kuo, and S. Granick, Bursts of Active Transport in Living Cells, Phys. Rev. Lett. 111, 208102 (2013).

[7] F. Höfling and T. Franosch, Anomalous transport in the crowded world of biological cells, Rep. Prog. Phys. 76, 046602 (2013).

[8] P. Habdas, D. Schaar, A. C. Levitt, and E. R. Weeks, Forced motion of a probe particle near the colloidal glass transition, Europhys. Lett. 67, 477 (2004).
[9] L. Wilson, A. Harrison, A. Schofield, J. Arlt, and W. Poon, Passive and active microrheology of hard-sphere colloids, J. Phys. Chem. B 113, 3806 (2009).

[10] D. Winter, J. Horbach, P. Virnau, and K. Binder, Active Nonlinear Microrheology in a Glass-Forming Yukawa Fluid, Phys. Rev. Lett. 108, 028303 (2012).

[11] C. J. Olson Reichhardt and C. Reichhardt, Fluctuations, jamming, and yielding for a driven probe particle in disordered disk assemblies, Phys. Rev. E 82, 051306 (2010).

[12] M. Gruber, G. C. Abade, A. M. Puertas, and M. Fuchs, Active microrheology in a colloidal glass, Phys. Rev. E 94, 042602 (2016).

[13] S. R. Williams and D. J. Evans, Linear Response Domain in Glassy Systems, Phys. Rev. Lett. 96, 015701 (2006).

[14] D. Winter and J. Horbach, Nonlinear active micro-rheology in a glass forming soft-sphere mixture, J. Chem. Phys. 138, 12A512 (2013).

[15] C. Reichhardt and C. J. Olson Reichhardt, Active microrheology in active matter systems: Mobility, intermittency, and avalanches, Phys. Rev. E 91, 032313 (2015).

[16] S.-H. Lee and D. G. Grier, Giant Colloidal Diffusivity on Corrugated Optical Vortices, Phys. Rev. Lett. 96, 190601 (2006).

[17] R. Candelier and O. Dauchot, Creep Motion of an Intruder Within a Granular Glass Close to Jamming, Phys. Rev. Lett. 103, 128001 (2009).

[18] D. L. Koch and J. F. Brady, Anomalous diffusion in heterogeneous porous media, Phys. Fluids 31, 965 (1988).

[19] J.-P. Bouchaud, A. Comtet, A. Georges, and P. Le Doussal, Classical diffusion of a particle in a one-dimensional random force field, Ann. Phys. (N.Y.) 201, 285 (1990).

[20] R. L. Jack, D. Kelsey, J. P. Garrahan, and D. Chandler, Negative differential mobility of weakly driven particles in models of glass formers, Phys. Rev. E 78, 011506 (2008).

[21] C. F. E. Schroer and A. Heuer, Anomalous Diffusion of Driven Particles in Supercooled Liquids, Phys. Rev. Lett. 110, 067801 (2013).

[22] G. Gradenigo, E. Bertin, and G. Biroli, Field-induced superdiffusion and dynamical heterogeneity, Phys. Rev. E 93, 060105 (2016).

[23] O. Bénichou, A. Bodrova, D. Chakraborty, P. Illien, A. Law, C. Mejía-Monasterio, G. Oshanin, and R. Voituriez, Geometry-Induced Superdiffusion in Driven Crowded Systems, Phys. Rev. Lett. 111, 260601 (2013).

[24] S. Leitmann and T. Franosch, Time-Dependent Fluctuations and Superdiffusivity in the Driven Lattice Lorentz Gas, Phys. Rev. Lett. 118, 018001 (2017).

[25] I. Gazuz, A. M. Puertas, T. Voigtmann, and M. Fuchs, Active and Nonlinear Microrheology in Dense Colloidal Suspensions, Phys. Rev. Lett. 102, 248302 (2009).

[26] C. Zhang, C. B. O'Donovan, E. I. Corwin, F. Cardinaux, T. G. Mason, M. E. Möbius, and F. Scheffold, Structure of marginally jammed polydisperse packings of frictionless spheres, Phys. Rev. E 91, 032302 (2015).

[27] C. Zhang, N. Gnan, T. G. Mason, E. Zaccarelli, and F. Scheffold, Dynamical and structural signatures of the glass transition in emulsions, J. Stat. Mech. (2016) 094003.

[28] T. G. Mason et al., Monodisperse emulsions: properties and uses, in Encyclopedia of EmulsionTechnology, edited by P. Becher (Marcel Dekker, NewYork, 1996), Vol. 4, p. 299. 
[29] A. J. Liu and S. R. Nagel, The jamming transition and the marginally jammed solid, Annu. Rev. Condens. Matter Phys. 1, 347 (2010).

[30] I. Jorjadze, L.-L. Pontani, and J. Brujic, Microscopic Approach to the Nonlinear Elasticity of Compressed Emulsions, Phys. Rev. Lett. 110, 048302 (2013).

[31] See Supplemental Material at http://link.aps.org/ supplemental/10.1103/PhysRevLett.122.108002, for additional text on methods and figures.

[32] C. P. Royall, W. C. Poon, and E. R. Weeks, In search of colloidal hard spheres, Soft Matter 9, 17 (2013).

[33] K. W. Desmond and E. R. Weeks, Influence of particle size distribution on random close packing of spheres, Phys. Rev. E 90, 022204 (2014).

[34] J.-Z. Xue, D. J. Pine, S. T. Milner, X.-1. Wu, and P. M. Chaikin, Nonergodicity and light scattering from polymer gels, Phys. Rev. A 46, 6550 (1992).

[35] G. L. Hunter and E. R. Weeks, The physics of the colloidal glass transition, Rep. Prog. Phys. 75, 066501 (2012).

[36] W. van Megen, T. C. Mortensen, S. R. Williams, and J. Müller, Measurement of the self-intermediate scattering function of suspensions of hard spherical particles near the glass transition, Phys. Rev. E 58, 6073 (1998).

[37] M. Sperl, Nearly-logarithmic decay in the colloidal hardsphere system, Phys. Rev. E 71, 060401 (2005).
[38] J. C. Crocker, J. A. Matteo, A. D. Dinsmore, and A. G. Yodh, Entropic Attraction and Repulsion in Binary Colloids Probed with a Line Optical Tweezer, Phys. Rev. Lett. 82, 4352 (1999).

[39] M. Woerdemann, in Structured Light Fields (Springer, Berlin Heidelberg New York, 2012), pp. 5-26.

[40] T. M. Aminabhavi and B. Gopalakrishna, Density, viscosity, refractive index, and speed of sound in aqueous mixtures of $n$, n-dimethylformamide, dimethyl sulfoxide, n, n-dimethylacetamide, acetonitrile, ethylene glycol, diethylene glycol, 1, 4-dioxane, tetrahydrofuran, 2-methoxyethanol, and 2-ethoxyethanol at 298.15 k, J. Chem. Eng. Data 40, 856 (1995).

[41] P. Sharma, S. Ghosh, and S. Bhattacharya, A high-precision study of hindered diffusion near a wall, Appl. Phys. Lett. 97, 104101 (2010).

[42] H. Risken, Fokker-planck equation, in The Fokker-Planck Equation (Springer, Berlin Heidelberg New York, 1996), pp. 163-178.

[43] L. S. Froufe-Pérez, M. Engel, J. J. Sáenz, and F. Scheffold, Band gap formation and Anderson localization in disordered photonic materials with structural correlations, Proc. Natl. Acad. Sci. U.S.A. 114, 9570 (2017).

[44] E. R. Weeks, J. C. Crocker, A. C. Levitt, A. Schofield, and D. A. Weitz, Three-dimensional direct imaging of structural relaxation near the colloidal glass transition, Science $\mathbf{2 8 7}$, 627 (2000). 